\title{
Quantitative scanning spreading resistance microscopy on n-type dopant diffusion profiles in germanium and the origin of dopant deactivation
}

Cite as: J. Appl. Phys. 125, 085105 (2019); https://doi.org/10.1063/1.5066617

Submitted: 16 October 2018 . Accepted: 09 February 2019 . Published Online: 27 February 2019

Jan K. Prüßing (D), Gerry Hamdana, Dominique Bougeard, Erwin Peiner, and Hartmut Bracht
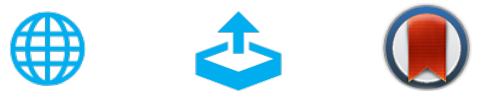

\section{ARTICLES YOU MAY BE INTERESTED IN}

Physics of picosecond pulse laser ablation

Journal of Applied Physics 125, 085103 (2019); https://doi.org/10.1063/1.5080628

Evolution of the light sensitive defects in high performance multicrystalline silicon wafers Journal of Applied Physics 125, 085701 (2019); https://doi.org/10.1063/1.5079496

Quantum well action model for the formation of a single Shockley stacking fault in a $4 \mathrm{H}-\mathrm{SiC}$ crystal under non-equilibrium conditions

Journal of Applied Physics 125, 085705 (2019); https://doi.org/10.1063/1.5074150

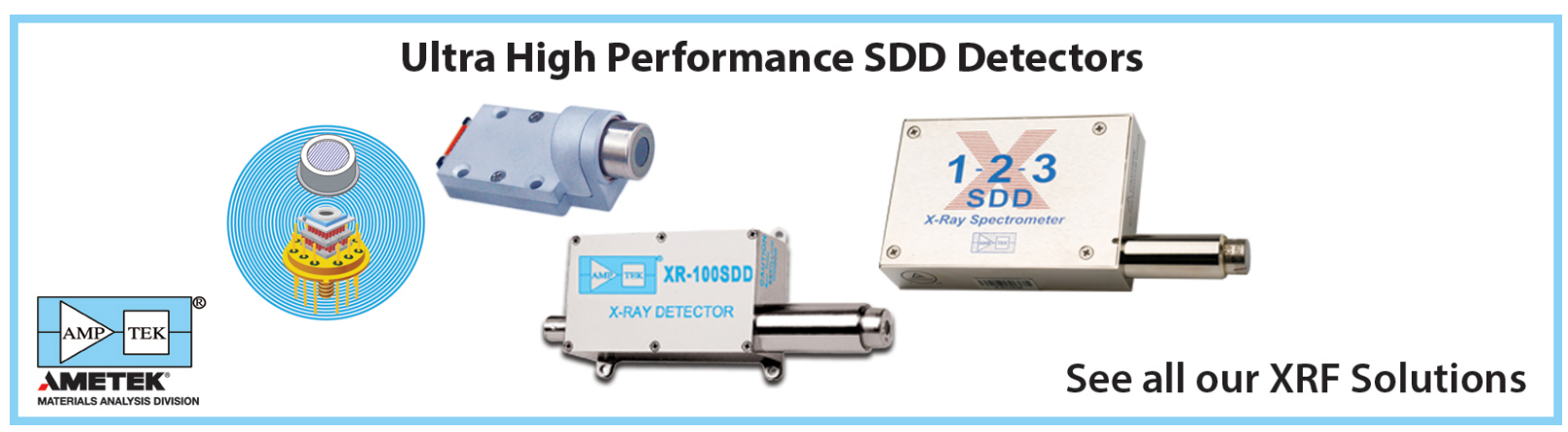




\title{
Quantitative scanning spreading resistance microscopy on $\mathrm{n}$-type dopant diffusion profiles in germanium and the origin of dopant deactivation
}

\author{
Cite as: J. Appl. Phys. 125, 085105 (2019); doi: 10.1063/1.5066617 \\ Submitted: 16 October 2018 - Accepted: 9 February 2019 . \\ Published Online: 27 February 2019
}

Jan K. Prüßing, ${ }^{1, \text { a) }}$ (D) Gerry Hamdana, ${ }^{2}$ Dominique Bougeard, ${ }^{3}$ Erwin Peiner, $^{2}$ and Hartmut Bracht ${ }^{7, b)}$

\author{
AFFILIATIONS \\ ${ }^{1}$ Institute of Materials Physics, University of Münster, Wilhelm-Klemm-Straße 10, D-48149 Münster, Germany \\ ${ }^{2}$ Institute of Semiconductor Technology (IHT) and Laboratory of Emerging Nanometrology (LENA), Technische Universität \\ Braunschweig, Hans-Sommer-Straße 66, D-38106 Braunschweig, Germany \\ ${ }^{3}$ Institute of Experimental and Applied Physics, University of Regensburg, D-93040 Regensburg, Germany \\ a)jan.pruessing@wwu.de \\ b) bracht@wwu.de
}

\begin{abstract}
Diffusion profiles of arsenic and antimony in undoped and carbon doped germanium (Ge), respectively, were analysed by means of scanning spreading resistance microscopy (SSRM). Whereas earlier secondary ion mass spectrometry analyses have determined the distribution of the chemical concentration of dopants and carbon, the electrically active defect concentration is quantified by SSRM using appropriate calibration samples and a preparation technique that reduces the surface roughness and its density of electronic states. Pronounced differences between the chemical and electrical dopant profiles are observed and consistently described by the formation of inactive dopant defect complexes in the framework of the vacancy mediated diffusion of donor atoms in Ge. This reveals that donor deactivation occurs during dopant diffusion at elevated temperatures.
\end{abstract}

Published under license by AIP Publishing. https://doi.org/10.1063/1.5066617

\section{INTRODUCTION}

One of the most important developments in the semiconductor industry during the past 50 years was the enormous downscaling of semiconductor devices, i.e., metal-oxidesemiconductor (MOS) transistors, from several $\mu \mathrm{m}$ in the $1970 \mathrm{~s}^{1}$ down to a few $\mathrm{nm}$ in the present FinFET structures. ${ }^{2,3}$ Controlling the doping concentration with either $\mathrm{p}$ - or $\mathrm{n}$-type dopants is still crucial for the preparation of functional electronic devices. The characterization of the dopant concentration can be performed by several techniques. Secondary ion mass spectrometry (SIMS) was probably one of the most widely used techniques. To keep up with the downscaling, SIMS depth resolution was improved down to $1 \mathrm{~nm}$, while the lateral resolution stayed at about $80 \mathrm{~nm} .{ }^{4,5}$ Accordingly, SIMS is not applicable for the characterization of nanoscopic electronic devices due to its limited lateral resolution. On the other hand, atom probe tomography (APT) shows a sub-nm resolution in three dimensions and even enables analyses of modern transistor structures. However, the preparation of APT tips from samples under study is quite laborious, and the detection limit for foreign atoms is in the range of typically $10^{19} \mathrm{~cm}^{-3}$. ${ }^{6}$ Moreover, the three dimensional reconstruction of the sample tip can be affected by artefacts arising from complex electric field distributions. These limitations also demand the calibration of APT by other techniques and constrain its use as a standard technique for a straightforward and simple characterization of semiconductor structures.

What both SIMS and APT have in common is that they detect the total, chemical dopant concentration, i.e., no information about the amount of charge carriers, which affect the device performance, is accessible. In cases where dopants are considered to become deactivated, i.e., for highly n-type doped germanium $(\mathrm{Ge}),{ }^{7-9}$ SIMS and APT cannot provide direct evidence about the electrically active dopant concentration. 
For many years now, spreading resistance profiling (SRP) is a suitable technique to measure charge carrier concentrations. ${ }^{10}$ This technique is widely applied to determine carrier profiles associated with electrically active foreign atoms in $\mathrm{Ge}$ and silicon (Si) (see, e.g., Refs. 9 and 11-13). Carrier profiles measured by means of SRP after diffusion experiments under well defined conditions serve as input data for modelling diffusion and defect reactions in semiconductors to characterize the involved point defects. ${ }^{14-16}$ SRP is a two point electrical measuring technique. The current $I_{\text {spread }}$ flowing through two tungsten-carbide tips in contact with the sample is antiproportional to the specific resistance $\rho$ of the underlying material. The specific resistance is interrelated to the concentration $C_{\text {elec }}$ of electrically active dopants via

$$
\frac{U}{\mathrm{I}_{\text {spread }}}=\mathrm{R}_{\text {spread }}=\frac{k \cdot \rho}{4 a}=\frac{k}{4 a \cdot e \cdot \mu \cdot \mathrm{C}_{\text {elec }}},
$$

with the number of tips $k$, the contact radius $a$ of the tips, the mobility of charge carrier $\mu$, and the electron charge $e .{ }^{10}$ The robust SRP-tip with up to $1 \mu \mathrm{m}$ contact radius naturally limits the resolution of this method. The resolution is substantially improved by scanning spreading resistance microscopy (SSRM), which is an atomic force microscope (AFM) based technique that uses the AFM-tip as contact to the sample. ${ }^{13,17,18}$ Based on this technique, two dimensional profiling with subnanometer resolution is reported. ${ }^{19}$

Quantification of SSRM resistance data is still a challenge, but it is possible as reported by Clarysse et al. ${ }^{20}$ The measured total resistance $R$ is a sum of the tip resistance $R_{\text {tip }}$, spreading resistance $R_{\text {spread }}(\rho)$, and contact resistance $R_{\text {contact }}$ between tip and sample. The latter strongly depends on the surface preparation. The tip radius $a$ is only roughly estimated by the manufacturer and can change during measurement. In addition, surface states may influence the amount of detected charge carriers. ${ }^{20-22}$ Therefore, SSRM is more often used for qualitative $^{23-27}$ rather than for quantitative $\mathrm{e}^{22}$ analyses of dopant distributions in semiconductors. Quantified SSRM analyses of the active dopant level in conjunction with results on the total chemical dopant concentration provide valuable information about the level of electrical dopant activation or deactivation. ${ }^{9,11,20}$ A deactivation of dopants can occur in the course of thermal treatments after implantation ${ }^{9,28}$ and thermal diffusion of dopants. ${ }^{8,29}$

In this work, arsenic and antimony dopant diffusion profiles in undoped and carbon doped Ge, respectively, earlier investigated mainly by SIMS,$^{29}$ are additionally analysed with SSRM. A cross-sectional preparation, completed with a chemical-mechanical-polishing step, is applied to all samples for the SSRM analysis. The preparation leads to smooth surfaces with low concentrations of electrically active surface states. The impact of surface states on the SSRM results is analysed by COMSOL Multiphysics simulations. Differently phosphorous doped epitaxial Ge layers serve as a calibration sample to quantify the SSRM data, i.e., to determine the concentration of electrically active dopants.
A comparison of the measured active and chemical dopant concentration profiles with numerical simulations on the diffusion of these n-type dopants in Ge provides direct experimental evidence on the type of dopant-defect complexes responsible for donor deactivation in Ge.

\section{EXPERIMENTAL}

Three different Ge samples were prepared for SSRM analyses. Sample I consists of an epitaxial layer with four $200 \mathrm{~nm}$ thick layers of different phosphorus (P) concentrations between $4 \times 10^{17} \mathrm{~cm}^{-3}$ and $1.5 \times 10^{19} \mathrm{~cm}^{-3}$. The epitaxial layer was grown by molecular beam epitaxy (MBE) on a (100)-oriented Ge substrate. The P concentration was determined by SIMS. Full electrical activation of the dopant can be assumed at this concentration range. Sample I acts as an n-type calibration sample. Sample II represents a Ge sample that was doped with arsenic (As) by diffusion annealing at $650^{\circ} \mathrm{C}$ for 155 min. For the diffusion experiment, a (100)-oriented p-type Ge sample with a thickness of about $500 \mu \mathrm{m}$ and resistivity $>35 \Omega \mathrm{cm}$ was evacuated in a quartz ampoule together with an As source. ${ }^{29}$ Sample III is characterized by five MBE-grown Ge layers highly doped with carbon $(\mathrm{C})$ and sandwiched between undoped natural Ge layers. Antimony (Sb) was diffused into the sample by closed ampoule annealing at $700^{\circ} \mathrm{C}$ for $90 \mathrm{~min}$. To stop the diffusion process, samples II and III were cooled to room temperature by quenching the diffusion ampoule in ethylene glycol. More detailed information about the As- and Sb-doped samples is given by Brotzmann et al. ${ }^{29}$

Different grinding and polishing steps were applied to prepare the samples for SSRM analyses. The Ge samples were mounted to a $90^{\circ}$-holder, and additional dummy Ge pieces were glued to the front surface using Gatan Epoxy to avoid bevelling of the sample edge. The sample cross section was ground using $\mathrm{Al}_{2} \mathrm{O}_{3}$ dissolved in $\mathrm{H}_{2} \mathrm{O}$ on a glass plate $(30 \mu \mathrm{m}$, $15 \mu \mathrm{m}, 5 \mu \mathrm{m}$, and $1 \mu \mathrm{m})$ and subsequently polished using diamond paste on an acrylic glass plate $(1 \mu \mathrm{m}$ and $0.25 \mu \mathrm{m})$. Finally, chemical-mechanical-polishing (CMP) was performed for 5 min using Köstrosol 3550, an aqueous dispersion of 35 $\mathrm{nm}$ amorphous Si dioxide nanoparticles, on a PT Super Plan Perforiert disk from Presi. The polished surface was electrically contacted using an eutectic indium-gallium alloy. The final surface roughness was determined by AFM non-contact topography measurement to be around $0.5 \mathrm{~nm}$ RMS.

SSRM measurements were performed on a Park XE-100 AFM using highly doped diamond coated DDESP-V2 tips from Bruker in contact mode at a force of $7 \mu \mathrm{N}$. A bias of $\mathrm{U}=+100 \mathrm{mV}$ was applied to the samples. Current I was measured by a DLPCA-200 logarithmic amplifier of Femto. SIMS measurements were performed with a Cameca system using oxygen as primary ion beam. ${ }^{29}$

\section{RESULTS AND DISCUSSION}

Sample I was analysed with both SIMS and SSRM. Resistance profiles extracted from the SSRM scan and the $\mathrm{P}$-concentration profile from the SIMS analysis are illustrated 
in Fig. 1. 1D profiles were extracted from the 2D SSRM scan (upper and lower right corners in Fig. 1) by taking the mean value of 128 line scans (the median value of 12 line scans with the highest current data) for the CMP treated sample (for the sample without CMP treatment). Note, the SSRM resistance decreases toward the top of the left ordinate. In this representation, a decreasing resistance corresponds to an increasing dopant concentration. SSRM analyses were performed with the same tip on samples prepared with (solid line) and without (long dashed line) a final CMP step. It is evident from Fig. 1 that a surface treatment with CMP reduces the measured resistance (solid line) within the P-doped region (below $1300 \mathrm{~nm}$ ) significantly. On the other hand, the resistance for the region above $1300 \mathrm{~nm}$ shows a higher resistance with CMP treatment than without. This impact of the sample preparation technique on the SSRM resistance data is explained by differences in the surface quality. Without a CMP treatment, the Ge surface is relatively rough $(1.3 \mathrm{~nm}$ RMS) compared to the roughness after CMP (0.5 $\mathrm{nm}$ RMS).

One consequence of a rough surface is a bad electrical contact between tip and sample. A good contact, i.e., with low resistance, is achieved by the formation of a metallic $\beta$-tin Ge phase underneath the probe. ${ }^{22}$ The phase transformation from diamond structure to $\beta$-tin starts at high pressures around $10 \mathrm{GPa}^{30}$ In the case of a rough surface, there is no or only a small area where the local pressure exhibits the pressure threshold, i.e., the overall dopant independent resistance $R_{0}$ increases and the effective contact radius $a$, which is
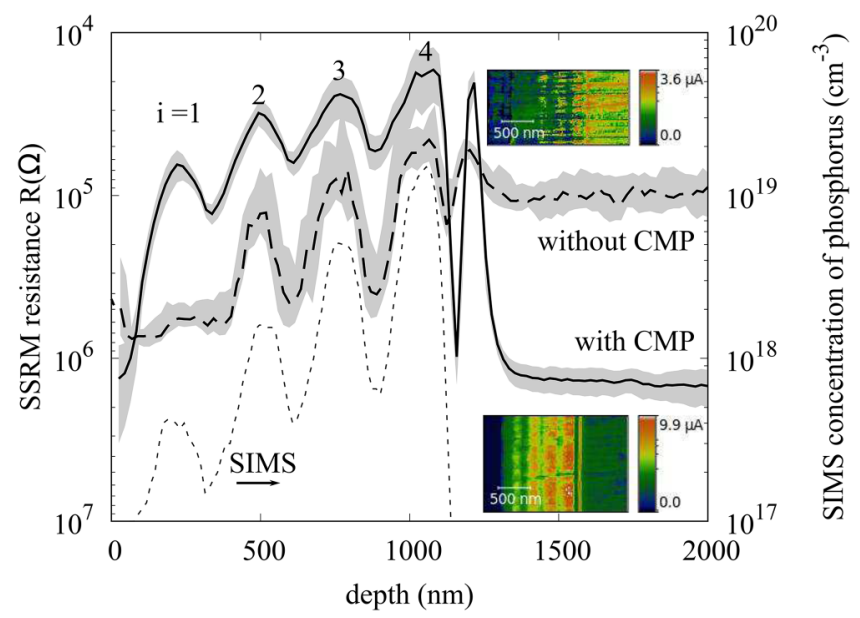

FIG. 1. SSRM cross-section resistance data (solid and long dashed lines) and SIMS concentration depth profile (short dashed line) of four P-doped layers epitaxially grown on a lowly doped Ge substrate (sample I). Variation in SSRM data is indicated by the grey band. The Ge substrate is located at depths $x>1300 \mathrm{~nm}$. SSRM sample preparation was performed with (solid line) and without (long dashed line) chemical-mechanical polishing (CMP). Note, the SSRM resistance decreases toward the top of the ordinate. The corresponding 2D SSRM scans are presented in the upper (without CMP) and lower (with $\mathrm{CMP}$ ) right corners. This P-doped Ge structure serves as a calibration sample to convert the SSRM resistance to donor concentrations. mainly affected by the size of the $\beta$-tin phase ${ }^{31}$ decreases. This leads to a higher spreading resistance $R_{\text {spread }}$ [Eq. (1)]. Both effects cause a higher measured resistance $R$ that is observed in Fig. 1 for the sample without CMP for depths $<1300 \mathrm{~nm}$. An ineffective formation of $\beta$-tin phase underneath the probe cannot explain the lower resistance of the sample without CMP treatment at depths $>1300 \mathrm{~nm}$ compared to the CMP treated sample within this intrinsic Ge substrate regime with an expected high resistivity (see Fig. 1). The disparity is explained by Ge surface states, whose concentration can significantly affect SSRM analyses of $\mathrm{Ge},{ }^{22}$ in particular, when the surfaces are not well polished. Ge surface states are known to be $\mathrm{p}$-type ${ }^{20}$ and lead to hole conduction even for intrinsic Ge due to an accumulation of holes at the surface. ${ }^{21}$

The values for the resistances $R^{i}$ measured at the four positions of the P-doped layers (see Fig. 1) are shown in Fig. 2 vs. the specific resistance $\rho^{i}$. The specific resistance is obtained from the measured SIMS concentration at the particular peak taking into account the correlation between the n-type doping concentration and the specific resistance reported by Cuttriss. ${ }^{32}$ The SSRM resistance vs. specific resistance is accurately described with the linear relationship

$$
\mathrm{R}=m \cdot \rho+\mathrm{R}_{0}
$$

The parameters $m$ and $R_{0}$ determined from a least-square-fit algorithm are given in Fig. 2 for treatments with and without CMP. The data-point for the lowest P-concentration $\left(4 \times 10^{-17} \mathrm{~cm}^{-3}\right)$ was not considered for the calibration curve representing the without CMP case (filled circles) since otherwise the curve would strongly deviate from the expected linear behaviour. From a physical point of view, the deviation

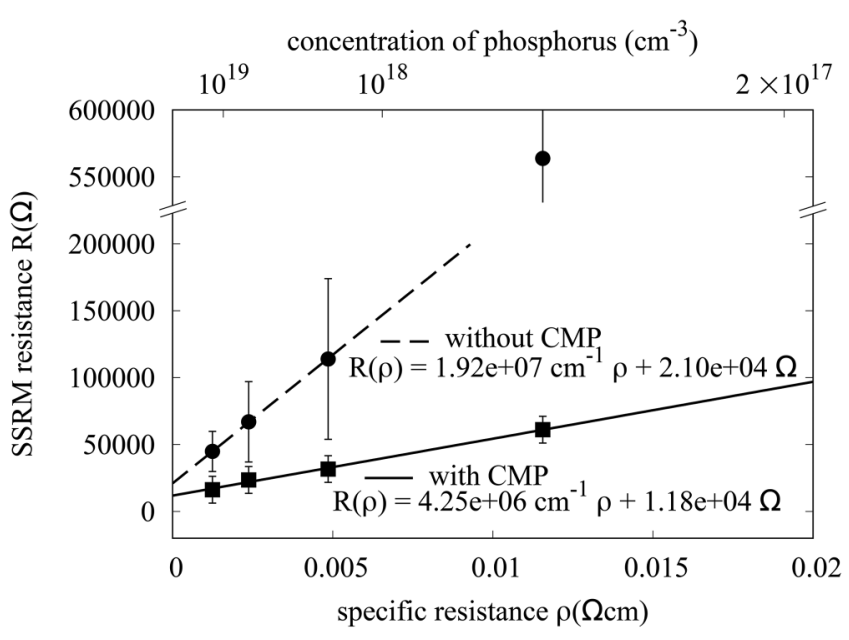

FIG. 2. SSRM resistance versus the specific resistance of the peak values from Fig. 1. Data are described by a linear $R(\rho)$-relation (solid and long dashed lines). The parameters of the best fit are indicated in the figure for the n-type germanium calibration sample I treated with $(\boldsymbol{\square})$ and without $(\bullet)$ CMP. 
from the linear behaviour corresponds to a deviation from the ideal case where Eq. (1) is valid (infinite sample volume, ohmic contact, no surface states).

The resistance parameter $R_{0}$ of Eq. (2) decreases from $21 \mathrm{k} \Omega$ for the untreated to $12 \mathrm{k} \Omega$ for the CMP treated sample. The resistance $R_{\text {tip }}$ of the doped conductive coating of the AFM tip is about $10 \mathrm{k} \Omega$ as measured on a gold surface. The close agreement to the $R_{0}$ value of the CMP treated calibration sample shows that other contributions to $R_{0}$ such as $R_{\text {contact }}$ can be neglected for CMP treated samples. The slope of the calibration curves in Fig. 2 decreases from $19.2 \times 10^{6} \mathrm{~cm}^{-1}$ to $4.3 \times 10^{6} \mathrm{~cm}^{-1}$ for CMP treated surfaces (solid line). This indicates an increase in the contact radius $a$ [see Eq. (1)] from $0.15 \mathrm{~nm}$ to $0.6 \mathrm{~nm}$. Both contact radii are much smaller than the expected tip radius of around $10 \mathrm{~nm}$ that is in accordance with MD simulations of SSRM tip-contacts on $\mathrm{Si}^{31}$ Obviously, the CMP treatment affects the effective contact radius.

Without the CMP treatment, only three SSRM data corresponding to P-concentrations $>10^{18} \mathrm{~cm}^{-3}$ are described by Eq. (2) (see dashed line in Fig. 2). For the lowest $\mathrm{P}$-concentration, the SSRM value is increased and strongly deviates from the linear $R(\rho)$ relationship. This increase in resistance is explained by recombination of surface states induced holes with free electrones. ${ }^{33}$ The resulting decreasing concentration of charge carriers is mostly pronounced for lowly n-type doping and explains the strong increase of the resistance value of peak $i=1$ for the sample without CMP compared to the treatment with CMP. Alternatively, the increased resistance at this peak could indicate a rectifying contact between tip and sample as reported by Schulze. ${ }^{34}$ Band bending leads to a potential barrier for positive sample bias. In the case of high doping concentrations, the barrier can easily be overcome by tunneling of charge carriers. In the case of low doping, an increased resistance with respect to the linear $\mathrm{R}(\rho)$-relation is expected.

With CMP, all four calibration data are accurately described by a linear $R(\rho)$ relation [see Eq. (2)]. This proves that the CMP treatment of Ge allows us to perform SSRM analyses of donor concentrations down to $10^{17} \mathrm{~cm}^{-3}$ without any significant impact of surface states or a Schottky contact barrier.

To further analyse a possible impact of surface states on our measurements, a three-dimensional simulation of SSRM was performed utilizing the COMSOL Multiphysics Semiconductor Module. The software solves Poisson- and continuity-equations for both electrons and holes under various boundary conditions. Figure 3(a) displays the simulation cell consisting of a Ge cuboid with a Ge-oxide surface and an ohmic back-contact at positive bias U. A round ohmic contact with radius $a$ is chosen as tip contact that can be placed at different positions. The P-concentration of sample I determines the donor concentration inside the simulation cell. P-type surface states with energy levels $100 \mathrm{meV}$ above the valence band edge are considered at the interface between $\mathrm{Ge}$ and the oxide. ${ }^{22}$

Figure 3(b) compares the experimental SSRM data of sample I (grey band) with calculated resistance profiles. The (a)
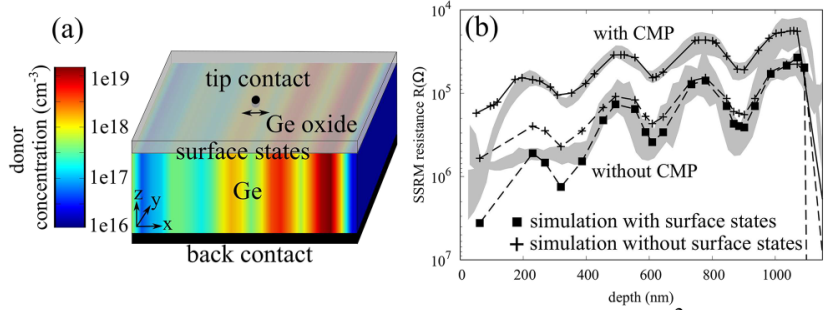

concentration of phosphorus $\left(\mathrm{cm}^{-3}\right)$

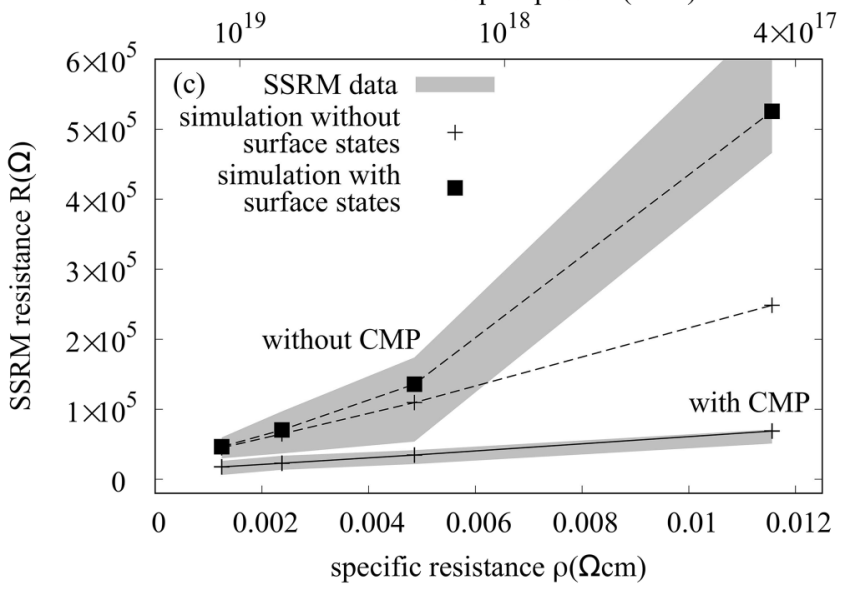

FIG. 3. (a) SSRM simulations performed by means of the COMSOL Multiphysics semiconductor module assuming a three-dimensional simulation cell with the P-concentration depth profile of sample I. (b) Calculated SSRM resistances $(\boldsymbol{\square}$ and +$)$ for different depths are shown in comparison to the SSRM measurements (grey bands) of calibration sample I prepared with and without CMP. A contact radius $a=0.6 \mathrm{~nm}(a=0.15 \mathrm{~nm})$ and a resistance $R_{0}=12 \mathrm{k} \Omega\left(R_{0}=21 \mathrm{k} \Omega\right)$ were considered for simulations of SSRM measurements on samples treated with (without) CMP. The calculated SSRM profile for sample I with CMP treatment (see topmost crosses) accurately reproduces the experimental result (see upper grey band). The simulation of the resistance profile for sample I without CMP treatment can only describe the experimental data [lower grey band in (b)] in the case when surface states at the $\mathrm{Ge} /$ Ge-oxide interface are taken into account [see $\mathbf{D}$ in (b)]. Simulated calibration curves are shown in (c) in comparison to the experimental results (upper and lower grey band). The maximum resistance at each peak with their specific resistance was considered for this representation.

SSRM profile obtained after the CMP treatment (upper grey band) is accurately reproduced by simulations (upper crosses) assuming a sample bias $U=+100 \mathrm{mV}$, a contact radius $a=0.6 \mathrm{~nm}$, no surface states, and a dopant independent tip resistance of $R_{\text {tip }}=R_{0}=12 \mathrm{k} \Omega$. The latter parameter is simply added to the calculated resistance values. The experimental SSRM profile of the sample without CMP treatment (lower grey band) and, in particular, the resistance range for low dopant concentrations $<10^{18} \mathrm{~cm}^{-3}$ is not well reproduced by the calculations neglecting surface states [lower crosses in Fig. 3(b)]. With surface states of $6 \times 10^{11} \mathrm{~cm}^{-2}$, the calculations (black squares) describe the experimental profile fairly well. The simulation parameters are $\mathrm{U}=+100 \mathrm{mV}, a=0.15 \mathrm{~nm}$, and $R_{0}=21 \mathrm{k} \Omega$. The settings account for a roughness related 
decreasing contact radius and an increasing contact resistance. The overall good agreement between the calculated and measured SSRM data is evident by the simulated calibration curves shown in Fig. 3(c) in comparison to the experimental SSRM results (grey band). The simulations with (without) surface states, which are indicated by black squares (crosses), accurately describe (strongly deviate from) the experimental SSRM data of sample I without the CMP treatment. On the other hand, the SSRM data of sample I with CMP (lower grey band) are closely reproduced by SSRM simulations without a significant contribution of surface states. The simulations support the impact of surface states on SSRM in the case of rough sample surfaces. A more complex model that includes trap-assisted tunnelling and a Schottky tip contact is reported by Schulze et $a .^{22}$ to accurately describe SSRM especially for lower dopant concentrations.

In order to detect possible changes in the calibration curve due to changes in the tip geometry in the course of the SSRM measurement, the SSRM analysis of calibration sample I is conducted right before and after the analyses of samples II and III. Both samples were prepared with a final CMP step.

Figure 4(a) shows the total As concentration profile of sample II measured by SIMS (plus symbols) and the corresponding profile of the electrically active dopant obtained by SSRM (crosses). The chemical concentration profile of As was originally measured by Brotzmann et al. ${ }^{29}$ The displayed 1D electrically active profile was extracted from a 2D SSRM scan [see insert of Fig. 4(a)] by taking the mean value of 128 line scans. The profiles clearly deviate for penetration depths below $800 \mathrm{~nm}$. Whereas the SSRM profile is rather flat with a maximum concentration of $2 \times 10^{19} \mathrm{~cm}^{-3}$, the SIMS concentration at the surface is $4 \times 10^{19} \mathrm{~cm}^{-3}$ and decreases continuously. ${ }^{35}$ On the other hand, Fig. 4(b) illustrates the total concentration of $\mathrm{Sb}$ (plus symbols) and $\mathrm{C}$ (dotted line) versus penetration depth recorded with SIMS by Brotzmann et al..$^{29}$ and the carrier concentration due to Sb doping measured with SSRM (crosses) of sample III. The 1D electrically active profile was extracted from the 2D SSRM scan [see the inset of Fig. 4(b)] by taking the mean value of 128 line scans. On first sight, the SSRM and SIMS profiles clearly deviate, i.e., the maxima of the SSRM coincide with the minima of the SIMS profile and the maxima in the SIMS profile convert to minima in the SSRM. This correlation of the SSRM and SIMS profiles suggests that carbon doping affects not only the amount of Sb incorporated in Ge but also the resistivity. An increased total $\mathrm{Sb}$ concentration is detected with SIMS for C concentrations of $\approx 10^{20} \mathrm{~cm}^{-3} \mathrm{com}-$ pared to regions with lower $C$ concentrations of $\approx 10^{18} \mathrm{~cm}^{-3}$. On the other hand, SSRM suggests a lower resistivity and thus seemingly higher concentrations of active dopants within the low-carbon doped compared to the high-carbon doped regions. Furthermore, SIMS detects sharp Sb- and $\mathrm{C}$-concentration peaks at $1400 \mathrm{~nm}$, while SSRM reveals a broad peak at this depth.

In order to evaluate the accuracy of the SSRM profiles, the chemical and electrical diffusion profiles of As and
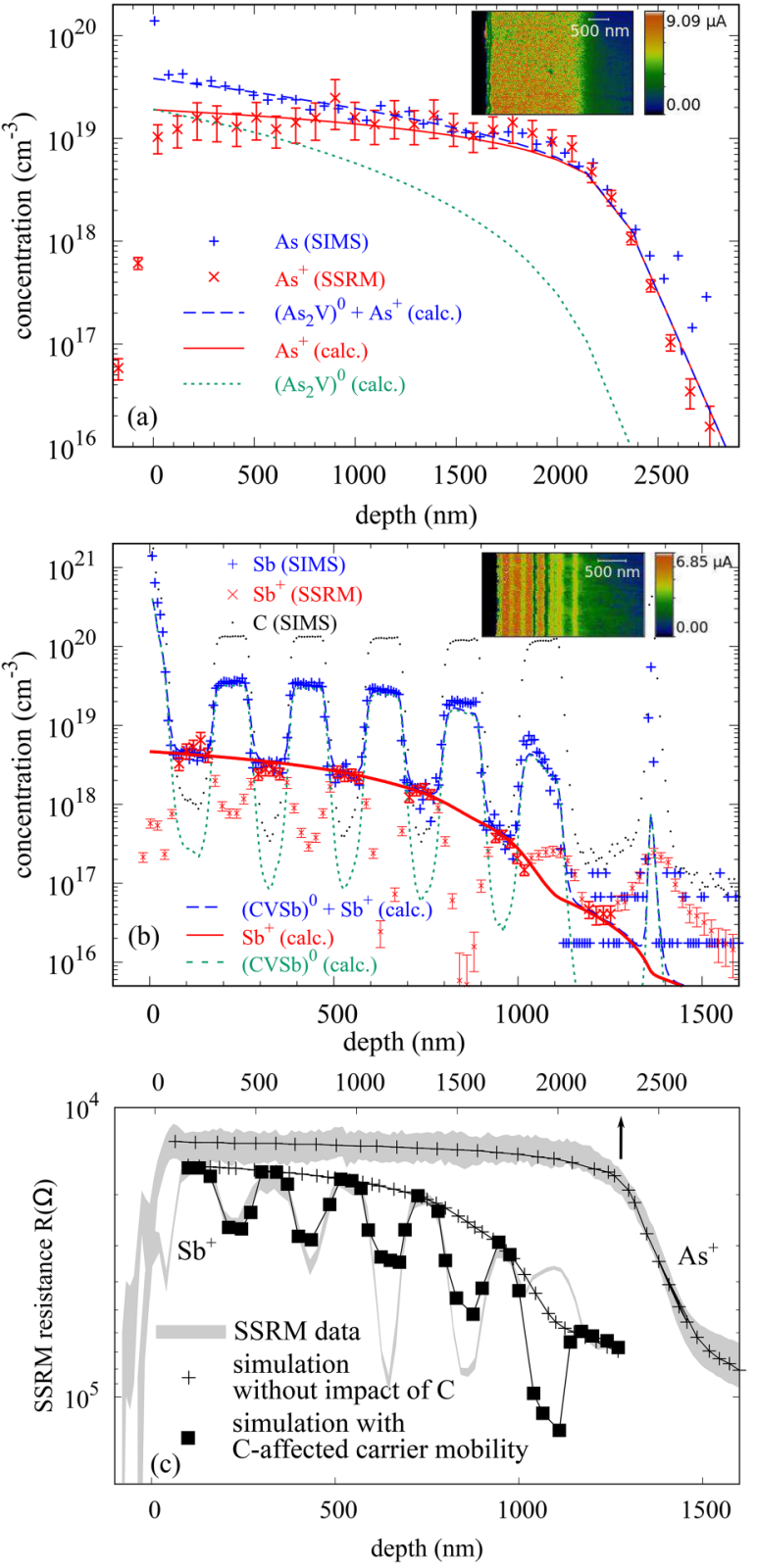

FIG. 4. Comparison of SSRM data $(x)$ and SIMS data $(+)$ with simulated concentration profiles of (a) electrically active $\mathrm{As}^{+}$(red solid line), neutral $\left(\mathrm{As}_{2} \mathrm{~V}\right)^{0}$ (green short dashed line), and sum of both species (blue long dashed line) for As-diffusion in bulk $\mathrm{Ge}\left(650^{\circ} \mathrm{C}, 155 \mathrm{~min}\right.$, sample II) and (b) electrically active $\mathrm{Sb}^{+}$ (red solid line), neutral (CVSb) ${ }^{0}$ (green short dashed line), and sum of both species (blue long dashed line) for Sb-diffusion profile $\left(700^{\circ} \mathrm{C}, 90 \mathrm{~min}\right.$, sample III) in five C-doped epi-layers on a lowly doped Ge substrate (starting at $1400 \mathrm{~nm}$ depth). The carbon profile in (b) is indicated by black circles. (c) Comparison of experimental SSRM data of $\mathrm{As}^{+}$(upper grey band) and $\mathrm{Sb}^{+}$(lower grey band) to SSRM profiles calculated by means of COMSOL Multiphysics with $(\boldsymbol{\square})$ and without $(+)$ an impact of carbon on the carrier mobility. Simulation parameters are $a=1.4 \mathrm{~nm}(a=1.6 \mathrm{~nm})$ for $\mathrm{As}^{+}\left(\mathrm{Sb}^{+}\right), R_{0}=11 \mathrm{k} \Omega$, and $U=100 \mathrm{mV}$. Note, the SSRM profile of $\mathrm{As}^{+}$is referred to the upper $\mathrm{x}$-scale. 
$\mathrm{Sb}$ in $\mathrm{Ge}$ are analysed in the framework of the vacancy diffusion mechanism

$$
(\mathrm{XV})^{-} \rightleftharpoons \mathrm{X}_{\mathrm{s}}^{+}+\mathrm{V}^{2-}
$$

where $(\mathrm{XV})^{-}, \mathrm{X}_{\mathrm{s}}^{+}$, and $\mathrm{V}^{2-}$ represent the singly negatively charged mobile dopant-vacancy pair, the singly positively charged substitutional donor atom, and the doubly negatively charged vacancy, respectively. This diffusion-reaction equation and the respective charge states of the point defects involved were determined from the concentration dependence of n-type dopant diffusion ${ }^{14,15,36,37}$ and their impact on Ge self-diffusion. ${ }^{29,38}$ The difference in the charge state of the mobile $(\mathrm{XV})^{-}$and immobile $\mathrm{X}_{\mathrm{s}}^{+}$defects results in a strong doping dependence of the donor diffusion coefficient $D_{X}$ that is described by $D_{X}=\left(n / n_{\text {in }}\right)^{2} D_{X}^{\text {in }},{ }^{39}$ where $D_{X}^{\text {in }}, n$, and $n_{\text {in }}$ represent the donor diffusion coefficient under electronically intrinsic conditions, the free electron concentration, and the carrier density under intrinsic conditions. The dependence of $D_{X}(X \in\{P, A s, S b\})$ on the square of $n$ accurately describes the diffusion profiles of substitutionally dissolved donor atoms $\mathrm{X}_{\mathrm{s}}^{+}$determined by means of SRP. ${ }^{14,15}$

Chemical concentration profiles of $\mathrm{X}$ measured with SIMS were reported by Brotzmann et $a .^{29}$ to be at variance to the distribution of electrically active donors predicted on the basis of the vacancy mechanism. The difference was proposed to be caused by the formation of neutral dopant-vacancy complexes $\left(\mathrm{X}_{2} \mathrm{~V}\right)^{0}$ via reaction ${ }^{29,36}$

$$
(\mathrm{XV})^{-}+\mathrm{X}^{+} \rightleftharpoons\left(\mathrm{X}_{2} \mathrm{~V}\right)^{0}
$$

The validity of reaction (4) for dopant diffusion in Ge is verified by a direct comparison between the chemical and electrical dopant profiles. A previous attempt to quantitatively compare the dopant profiles measured with SIMS and nano-SRP was hindered due to an insufficient accuracy and resolution of the electrical profiling technique at that time. ${ }^{29}$ The former SRP profile was obtained after Sb diffusion at $700{ }^{\circ} \mathrm{C}$ for $3 \mathrm{~h}$. This profile reveals a more sinusoidal shape than the SSRM profile shown in Figs. 4(b) and 4(c) that was measured after $\mathrm{Sb}$ diffusion at $700^{\circ} \mathrm{C}$ for $90 \mathrm{~min}$ into the same Ge structure. Since the SRP and the more recent SSRM measurements were conducted on samples annealed for different times, a direct comparison of the measurements is not meaningful and thus not shown in Fig. 4.

Additional proposed contributions to n-type dopant diffusion in Ge via neutral dopant-vacancy pairs ${ }^{40}$ and doubly negatively charged dopant-vacancy pairs ${ }^{41}$ could not be verified. Figure 4(a) displays the chemical and electrically active concentration of As in Ge after diffusion at $650{ }^{\circ} \mathrm{C}$ for $155 \mathrm{~min}$ (sample II) and compares the concentration profile of electrically active $\mathrm{As}_{\mathrm{s}}^{+}$determined with SSRM (crosses) to the $\mathrm{As}_{\mathrm{s}}^{+}$ profile (solid line) obtained from numerical calculations of As diffusion in Ge. The simulations are based on reactions (3) and (4). The measured and simulated $\mathrm{As}_{\mathrm{s}}^{+}$-profiles are in accurate agreement down to concentration of $10^{17} \mathrm{~cm}^{-3}$. The total
As concentration [long dashed line in Fig. 4(a)] comprises the concentrations of $\mathrm{As}_{\mathrm{s}}^{+}$and $\left(\mathrm{As}_{2} \mathrm{~V}\right)^{0}$ (short dashed line) and is in accurate agreement with the SIMS data (plus symbols). This agreement between the directly measured and predicted $\mathrm{As}_{\mathrm{s}}^{+}$ concentration profile not only confirms the diffusion and deactivation mechanisms of As in Ge expressed by reactions (3) and (4) but also reveals that the deactivation mainly occurs in the course of dopant diffusion and not during cooling of the sample to room temperature.

The significance of combined chemical and electrical profiling for the characterization of diffusion and defect reactions in Ge is also demonstrated by Fig. 4(b). This figure displays the measured SIMS and SSRM data of sample III that reveal a strong segregation of $\mathrm{Sb}$ in $\mathrm{C}$-rich regions after $\mathrm{Sb}$ diffusion in Ge at $700{ }^{\circ} \mathrm{C}$ for $90 \mathrm{~min}$. The concentration profile of the substitutional donor $\mathrm{Sb}_{\mathrm{s}}^{+}$(crosses) determined with SSRM is compared to diffusion simulations based on reactions (3) and (4) and the reaction

$$
(\mathrm{XV})^{-}+\mathrm{C}_{\mathrm{s}}^{0} \rightleftharpoons(\mathrm{CVX})^{0}+e^{-}
$$

that considers a segregation of $\mathrm{Sb}$ within the $\mathrm{C}$-doped region via the formation of neutral (CVSb) ${ }^{0}$ defect complexes. ${ }^{29}$ The previous diffusion simulations only aimed to accurately reproduce the chemical Sb concentration profile measured with SIMS (plus symbols) and yield predictions about the distribution and concentration of electrically active $\mathrm{Sb}_{\mathrm{s}}^{+}$and neutral $\left(\mathrm{Sb}_{2} \mathrm{~V}\right)^{0}$ and $(\mathrm{CVSb})^{0}$ complexes. $^{29}$ The calculated profiles of $\mathrm{Sb}_{\mathrm{s}}^{+}$and $(\mathrm{CVSb})^{0}$ are displayed in Fig. 4(b) by the solid and short dashed lines, respectively. The $\left(\mathrm{Sb}_{2} \mathrm{~V}\right)^{0}$ profile is not shown as its concentration is negligibly small compared to the concentrations of $\mathrm{Sb}_{\mathrm{S}}^{+}$and $(\mathrm{CVSb})^{0}{ }^{29}$ The total concentration of $\mathrm{Sb}$ shown in Fig. 4(b) by the long dashed line is mainly determined by $\mathrm{Sb}_{\mathrm{s}}^{+}$and $(\mathrm{CVSb})^{0}$. A comparison of the $\mathrm{Sb}_{\mathrm{s}}^{+}$ profile obtained by SSRM (crosses) with the calculated $\mathrm{Sb}_{\mathrm{s}}^{+}$ profile (solid line) reveals an accurate agreement for regions with low $\mathrm{C}$ concentration but a strong discrepancy for high $\mathrm{C}$ concentrations. This correlation between the electrical SSRM data and the $\mathrm{C}$ distribution is explained by the carrier mobility $\mu$ that is significantly reduced in regions with a high amount of $(\mathrm{CVSb})^{0}$ complexes. $^{29}$ With reference to Eq. (1), a change in $\mu$ also affects the measured spreading resistance $R_{\text {spread }}$. This is confirmed by the SSRM simulations presented in Fig. 4(c). The experimental SSRM resistance recorded after Sb diffusion in the C-doped Ge structure is fairly well reproduced by SSRM simulations, assuming that the carrier mobility $\mu$ is affected by high carbon concentrations $C_{C}$ in the layer structure using the following expression:

$$
\mu\left(C_{C}\right)=\mu_{0} \cdot\left(1+\sqrt{\frac{C_{C}}{C_{r e f}}}\right)^{-1},
$$

where $\mu_{0}$ represents the carrier mobility affected by the dopant concentration $C_{\text {elec }}$ and $C_{r e f}=2.5 \times 10^{19} \mathrm{~cm}^{-3}$ is a reference concentration. Neglecting the impact of carbon, only the measured SSRM resistance within the $\mathrm{C}$-undoped $\mathrm{Ge}$ 
layers is described. Correspondingly, the experimental SSRM resistance profile of the As-diffused and $\mathrm{C}$-undoped $\mathrm{Ge}$ sample II is also well reproduced by the SSRM simulations.

The sharp peak observed in Fig. 4(b) in the chemical Sb (plus symbol) and C (dotted line) concentration at about 1400 $\mathrm{nm}$ reveals a segregation at the interface of the Ge substrate and the epitaxially grown MBE layer. The SSRM peak at the interface is, in contrast, quite broadened. This is likely caused by interface states that affect the charge carrier concentration in its vicinity. The SSRM measurement (red crosses) reveals an interesting doping behaviour at depths of $1030 \mathrm{~nm}$ to $1150 \mathrm{~nm}$, i.e., at the position of the fifth $\mathrm{C}$-doped layer. This also becomes evident by Fig. 4(c) in the SSRM data (grey band) of the Sb-diffused sample III. The observed behaviour is at variance to the doping characteristic of the $\mathrm{C}$-doped regions at depths below $1000 \mathrm{~nm}$. Within the fifth C-doped Ge layer, the SSRM resistance is lower and suggests a higher active dopant concentration compared to the C-doped layers below $1000 \mathrm{~nm}$. It is also noticeable that the chemical $\mathrm{Sb}$ concentration within the fifth C-doped layer drops from about $10^{19} \mathrm{~cm}^{-3}$ down to $2 \times$ $10^{18} \mathrm{~cm}^{-3}$ with increasing depth [see Fig. 4(b)], whereas the active doping level even slightly increases from about $2 \times$ $10^{17} \mathrm{~cm}^{-3}$ to $3 \times 10^{17} \mathrm{~cm}^{-3}$. This could indicate that a trapping of $\mathrm{Sb}$ within the $\mathrm{C}$-doped Ge layers is initially not immediately linked to a deactivation of $\mathrm{Sb}$, i.e., the deactivation evolves in the course of the proceeding segregation process. This could indicate the formation of an electrical active intermediate defect that further evolves to a neutral defect complex. A small but non-negligible electrical activity of $\mathrm{C}$ within the highly $\mathrm{C}$-doped regions can be excluded as explanation because the SSRM analysis of the as-grown Ge structure shows a constant carrier profile representative for electronically intrinsic Ge. Additional studies on Sb diffusion and its activation in Ge structures with different $\mathrm{C}$ doping levels are required to clarify the peculiar impact of $\mathrm{C}$ on $\mathrm{Sb}$ doping.

\section{CONCLUSION}

We performed SSRM analyses of arsenic and antimony diffusion profiles in originally undoped and carbon-doped germanium, respectively. The electrical active donor concentration, which was obtained from a calibration of SSRM resistances to free carrier concentrations, was compared with the total chemical dopant concentration determined by SIMS in earlier experiments. Different sample preparation methods show the impact of surface roughness on SSRM resistance profiles. COMSOL Multiphysics simulations confirm the hypothesis that surface states are mainly responsible for the observed increase in the measured resistance for lowly n-doped regions. A chemical-mechanical polishing (CMP) surface treatment is applied as an effective preparation step to decrease the density of surface states. By means of this preparation method, we were able to detect electrically active $\mathrm{n}$-type dopant profiles in germanium with some $10 \mathrm{~nm}$ depth resolution and concentrations down to $10^{17} \mathrm{~cm}^{-3}$. The measured active As and $\mathrm{Sb}$ profiles are in accurate agreement with the diffusion profiles of substitutionally dissolved dopants calculated by numerical simulations on the basis of the vacancy mechanism (3) and additional reactions that consider the formation of dopant-vacancy (4) and dopantcarbon (5) complexes. The consistency between experimental and theoretical dopant profiles with regard to both the total chemically and electrically active concentration not only shows the validity of reactions (3)-(5) for the diffusion of arsenic and antimony in Ge but also reveals the significance of neutral dopant-vacancy $\left(\mathrm{X}_{2} \mathrm{~V}\right)^{0}$ and neutral carbon-defect $(\mathrm{CVSb})^{0}$ complexes that form in the course of diffusion in originally undoped and carbon-doped germanium, respectively. Moreover, the work shows the potential of combined chemical and electrical analyses of dopant diffusion profiles for the characterization of diffusionreaction processes in semiconductors. The atomic mechanisms derived from such studies are fundamental, i.e., will also hold for semiconductor structures with a high surface-to-volume ratio as long as the physical properties of the semiconductor structure equal the properties of the bulk material. However, dopant diffusion and activation in nanoscopic structure can be affected by surface states that can pin the Fermi level and give rise to band bending. As a consequence, the formation enthalpy of charged point defects in nanoscopic structures will be affected and thus the defectmediated dopant diffusion.

\section{ACKNOWLEDGMENTS}

This work was funded by the Deutsche Forschungsgemeinschaft under Grant Nos. BR 1520/16-1, PE 885/3-1, and BO 3140/4-1.

\section{REFERENCES}

${ }^{\mathbf{1}}$ R. Kumar, Fabless Semiconductor Implementation (McGraw-Hill, New York, 2008).

${ }^{2}$ Z. Ma and D. G. Seiler, Metrology and Diagnostic Techniques for Nanoelectronics (Pan Stanford, 2017).

${ }^{3}$ S. Narasimha, B. Jagannathan, A. Ogino, D. Jaeger, B. Greene, C. Sheraw, K. Zhao, B. Haran, U. Kwon, A. K. M. Mahalingam et al., in 2017 IEEE International Electron Devices Meeting (IEDM) (IEEE, 2017).

${ }^{4}$ C. W. Magee, R. S. Hockett, T. H. Büyüklimanli, I. Abdelrehim, and J. W. Marino, Nucl. Instrum. Methods Phys. Res. B 261, 594 (2007)

${ }^{5}$ Iontof $\mathrm{GmbH}$ (2018), see https://www.iontof.com/download/IONTOF TOF-SIMS-5_Bi-Nanoprobe.pdf.

${ }^{6}$ T. F. Kelly, B. P. Geiser, R. M. Ulfig, T. J. Prosa, and D. J. Larson, Local Electrode Atom Probe Tomography: A User's Guide (Springer, 2013).

7J. Coutinho, C. Janke, A. Carvalho, V. Torres, S. Oberg, R. Jones, and P. Briddon, Physica B 401-402, 179 (2007).

${ }^{8}$ T. Kalliovaara, J. Slotte, I. Makkonen, J. Kujala, F. Tuomisto, R. Milazzo, G. Impellizzeri, G. Fortunato, and E. Napolitani, Appl. Phys. Lett. 109, 182107 (2016).

${ }^{9}$ R. Milazzo, G. Impellizzeri, D. Piccinotti, D. De Salvador, A. Portavoce, A. La Magna, G. Fortunato, D. Mangelinck, V. Privitera, A. Carnera, and E. Napolitani, Appl. Phys. Lett. 110, 011905 (2017).

${ }^{10}$ T. Clarysse, D. Vanhaeren, I. Hoflijk, and W. Vandervorst, Mater. Sci. Eng. R Rep. 47, 123 (2004).

${ }^{11}$ D. Pastor, H. H. Gandhi, C. P. Monmeyran, A. J. Akey, R. Milazzo, Y. Cai, E. Napolitani, R. M. Gwilliam, I. F. Crowe, J. Michel, L. C. Kimerling, A. Agarwal, E. Mazur, and M. J. Aziz, J. Appl. Phys. 123, 165101 (2018).

${ }^{12}$ W. L. Harrington, C. W. Magee, M. Pawlik, D. F. Downey, C. M. Osburn, and S. B. Felch, J. Vac. Sci. Technol. B 16, 286 (1998). 
${ }^{13}$ P. De Wolf, T. Clarysse, W. Vandervorst, and L. Hellemans, J. Vac. Sci. Technol. B 16, 401 (1998).

${ }^{14} \mathrm{H}$. Bracht and S. Brotzmann, Mater. Sci. Semicond. Process. 9, 471 (2006).

${ }^{15}$ S. Brotzmann and H. Bracht, J. Appl. Phys. 103, 033508 (2008).

${ }^{16}$ H. Bracht, N. A. Stolwijk, and H. Mehrer, Phys. Rev. B 52, 16542 (1995).

${ }^{17} \mathrm{P}$. D. Wolf, "Two-dimensional carrier profiling of semiconductor structures with nanometer resolution," Ph.D. dissertation (Katholieke Universiteit Leuven, 1998).

${ }^{18}$ P. DeWolf, T. Clarysse, W. Vandervorst, L. Hellemans, Ph. Niedermann, and W. Hänni, J. Vac. Sci. Technol. B 16, 355 (1998).

${ }^{19}$ L. Zhang, H. Tanimoto, K. Adachi, and A. Nishiyama, IEEE Electron Device Lett. 29, 799 (2008).

${ }^{20}$ T. Clarysse, P. Eyben, T. Janssens, I. Hoflijk, D. Vanhaeren, A. Satta, M. Meuris, W. Vandervorst, J. Bogdanowicz, and G. Raskin, J. Vac. Sci. Technol. B 24, 381 (2006).

${ }^{21}$ T. Hanrath, and B. A. Korgel, J. Phys. Chem. B 109, 5518-5524 (2005).

${ }^{22}$ A. Schulze, T. Hantschel, P. Eyben, A. Verhulst, R. Rooyackers, A. Vandooren, and W. Vandervorst, Ultramicroscopy 125, 18 (2013).

${ }^{23}$ D. Alvarez, J. Hartwich, M. Fouchier, P. Eyben, and W. Vandervorst, Appl. Phys. Lett. 82, 1724 (2003).

${ }^{\mathbf{2 4}}$ S. Kluth, D. Alvarez, S. Trellenkamp, J. Moers, S. Mantl, J. Kretz, and W. Vandervorst, J. Vac. Sci. Technol. B 23, 76 (2005).

${ }^{25}$ C. Celle, C. Mouchet, E. Rouviere, J.-P. Simonato, D. Mariolle, N. Chevalier, and A. Brioude, J. Phys. Chem. C 114, 760 (2009).

${ }^{26}$ H. Yamagiwa, S. Abo, F. Wakaya, M. Takai, T. Sakamoto, H. Tokioka, and N. Nakagawa, Appl. Phys. Lett. 89, 062101 (2006).

${ }^{27}$ S. Doering, R. Rudolf, M. Pinkert, H. Roetz, C. Wagner, S. Eckl, M. Strasser, A. Wachowiak, and T. Mikolajick, Microelectron. Reliab. 54, 2128 (2014).
${ }^{28}$ A. R. Peaker, V. P. Markevich, B. Hamilton, I. D. Hawkins, J. Slotte, K. Kuitunen, F. Tuomisto, A. Satta, E. Simoen, and N. V. Abrosimov, Thin Solid Films 517, 152 (2008).

${ }^{29}$ S. Brotzmann, H. Bracht, J. Lundsgaard Hansen, A. Nylandsted Larsen, E. Simoen, E. E. Haller, J. S. Christensen, and P. Werner, Phys. Rev. B 77, 235207 (2008).

${ }^{30}$ J. C. Jamieson, Science 139, 762 (1963).

${ }^{31}$ P. Eyben, F. Clemente, K. Vanstreels, G. Pourtois, T. Clarysse, E. Duriau,

T. Hantschel, K. Sankaran, J. Mody, and W. Vandervorst, J. Vac. Sci. Technol. B 28, 401-3274 (2010).

${ }^{32}$ D. Cuttriss, Bell Syst. Tech. J. 40, 509 (1961).

${ }^{33}$ S. Zhang, E. R. Hemesath, D. E. Perea, E. Wijaya, J. L. Lensch-Falk, and L. J. Lauhon, Nano Lett. 9, 3268-3274 (2009).

${ }^{34} \mathrm{~A}$. Schulze, "Two-and three-dimensional dopand and conductivity profiling in confined volumes," Ph.D. dissertation (Katholieke Universiteit Leuven, 2013).

${ }^{35}$ The data point close to the surface is not assigned to the As diffusion profile but to a GeAs alloy formed in the course of diffusion annealing.

${ }^{36}$ A. Chroneos, R. W. Grimes, B. P. Uberuaga, and H. Bracht, Phys. Rev. B 77, 235208 (2008).

${ }^{37}$ A. Chroneos and H. Bracht, Appl. Phys. Rev. 1, 011301 (2014).

${ }^{38}$ M. Naganawa, Y. Shimizu, M. Uematsu, K. M. Itoh, K. Sawano, Y. Shiraki, and E. E. Haller, Appl. Phys. Lett. 93, 191905 (2008).

${ }^{39}$ H. Bracht, Phys. Rev. B 75, 035210 (2007).

${ }^{40}$ C. O. Chui, K. Gopalakrishnan, P. B. Griffin, J. D. Plummer, and K. C. Saraswat, Appl. Phys. Lett. 83, 3275 (2003).

${ }^{41}$ T. Canneaux, D. Mathiot, J.-P. Ponpon, and Y. Leroy, Thin Solid Films $\mathbf{5 1 8}$ 2394 (2010). 